\title{
Intermediate non-Gaussian transport in plasma core turbulence
}

\author{
T. Hauff and F. Jenko \\ Max-Planck-Institut für Plasmaphysik, \\ EURATOM Association, 85748 Garching, Germany
}

S. Eule

Institute for Theoretical Physics, University of Münster, 48149 Münster, Germany

\begin{abstract}
Test particle transport in realistic plasma core turbulence (as described by nonlinear gyrokinetics) is investigated, focussing on the question whether and under what conditions the transport may become 'anomalous', i.e. super- or subdiffusive. While in the presence of stochastic fluctuations, the transport always becomes diffusive for large times, coherent flow components like zonal flows or poloidal drifts can induce non-Gaussian transport over large intermedate time spans. In order to understand the origin of these phenomena, a simple model employing stochastic potentials is used to complement the analysis based on gyrokinetic turbulence simulations.
\end{abstract}




\section{INTRODUCTION}

In the fusion community, it is common to portray turbulent transport in tokamaks and stellarators as a standard diffusive process - in spite of its advective nature. However, there also exist several investigations dealing with the possibility of super- or subdiffusive transport, an interesting scenario which should not (and cannot) be excluded a priori. Unfortunately, neither the conditions under which such 'anomalous diffusion' is expected to occur nor its physical origin are well understood at present. This is the reason why in the present work, we attempt to shed some new light on this old question, employing self-created pseudo-turbulent velocity fields as well as data from nonlinear gyrokinetic simulations for parameters which are typical for tokamak core turbulence.

There is a number of well known experimental findings which can be interpreted as evidence for the existence of non-Gaussian transport. Among these are, for instance, the dependence of transport on the system size in low confinement mode plasmas,[1] the observed rapid propagation of an induced perturbation, $[2,3]$ or the measurement of long-range temporal and radial correlations in the plasma edge.[4-6] Such observations are sometimes explained in terms of avalanches or self-organized criticality (SOC). Inspired by experimental (and theoretical) evidence for the existence of critical gradients, [7] the model of the 'stochastic sandpile' [8] has been developed, for example. It is possible to link numerical simulations of plasma turbulence to the running sandpile by allowing for a temporal evolution of the mean profiles (and the respective gradients which drive the turbulence). Some numerical results are consistent with SOC characteristics $[9,10]$, and superdiffusive transport has been found, e.g., for pressure-gradient-driven plasma turbulence and attributed to avalanche effects.[11, 12] The idea of a critical density gradient acting as a threshold for avalanche transport has also been included into the framework of continuous time random walks (CTRWs), being able to reproduce some of the basic phenomenology of anomalous (superdiffusive) transport scaling in the low confinement mode.[13, 14] However, despite the interesting nature of these results, it is far from evident that they carry over to plasma core turbulence in large fusion devices like ITER.[15] Most of the experimental results showing superdiffusive transport are achieved for the plasma edge, and to which degree the observation of a 'prompt' perturbation response is of relevance to the usual steady-state conditions is not clear.[16] Moreover, some of the assumptions underlying the 'sandpile' based numerical 
work are not neccessarily justified in the plasma core of larger fusion devices.

Various papers approach the question of anomalous transport from a different perspective. Instead of trying to reproduce or analyze experimental data, they work with more simple turbulence models or analytically given fields in order to study the transition from a diffusive to a superdiffusive regime in detail. An interesting attempt to understand the basic mechansims of superdiffusive particle transport is the detection of so-called 'chaotic jets'.[17-19] Here, the origin of superdiffusion has been ascribed to the existence of longliving bundles of orbits with coherent propagation, constituting an independent structure, a 'hidden order' with almost free motion within the sea of turbulence. The life-time of such 'jets' was found to be much longer than the ordinary 'clump lifetime' of particles with close initial conditions.[20] In Ref. [18], a model of 16 point vortices was introduced, and a connection between superdiffusion and the accumulation of small Lyapunov exponents was established. In Ref. [19], the same technique was applied to the more realistic model of 2D Hasegawa-Mima turbulence, and similar results where found (see also Ref. [21] where superdiffusion was found for small box sizes and large density gradients). However, it is not at all clear if such minimal models - often yielding more or less stationary vortex structures lead to a good representation of turbulence in fusion devices. It is therefore necessary to analyze data from nonlinear gyrokinetic simulations which are considered to be first-principles based. This approach was chosen for the first time in Ref. [22]. Here, it was shown that fluctuating vortices, even when advected with a constant drift velocity, always lead to diffusive behavior for long times. However, in this case, zonal flows - which are a possible source for superdiffusive transport - were rather weak. In an analytic approach, modelling a turbulent bath with a single zonal flow as a vortex chain inside a shear layer, superdiffusive transport was found in the 'stochastic layer' where the particles alternate between being trapped in a vortex and moving ballistically with the shear flow.[23, 24] Consequently, superdiffusion was interpreted as a result of the presence of coherent structures in a turbulent background.

In the present paper, we intend to go through a two-step process. In the first step (Sec. II), we would like to study test particle transport in random (pseudo-turbulent) fields created by superposition of plane waves. This enables us to have easy control over the field's statistical properties, and allows for the inclusion of additional effects like poloidal drifts and zonal flows which will turn out to be crucial for the existence of non-Gaussian transport regimes. Then, in a second step (Sec. III), we will compare these results with simulations using realistic 
turbulent fields taken from simulations with the turbulence code Gene.[25, 26] Gene is a gyrokinetic Vlasov code, solving the nonlinear gyrokinetic equations [27-29] on a fixed grid in five-dimensional phase space (plus time), keeping the (average) profile gradients fixed. Examining two examples, trapped electron mode (TEM) turbulence and ion temperature gradient (ITG) turbulence, where the effects of poloidal drifts and zonal flows are rather strong, we find that while the transport gets diffusive and Gaussian for large times, there may exist super- and subdiffusive regimes for intermediate times. Given the fact that these (radially local) gyrokinetic simulations are known to be fully consistent with nonlocal ones for sufficiently small values of the normalized ion gyroradius $\rho^{*}=\rho_{s} / a\left(\rho^{*} \lesssim 1 / 300\right)[30,31]$, large-scale devices are expected to be well represented by the data used here. We will close with a summary and some conclusions in Sec. IV.

\section{DIFFUSION IN RANDOM FIELDS}

\section{A. Some preliminaries}

Throughout this paper, we consider the $E \times B$ advection of ions as passive tracers in a plane perpendicular to the background magnetic field, where the corresponding spatial coordinates will be denoted as $\mathbf{x}=\left(x_{1}, x_{2}\right)=(x, y)$. In Sec. III, the fluctuating electrostatic potentials $\phi(\mathbf{x}, t)$ will be taken from simulations with the gyrokinetic turbulence code GENE $[25,26]$, whereas in the present section, they will be created by superposing a sufficiently large number of random harmonic waves:[22, 32]

$$
\phi(\mathbf{x}, t)=\sum_{i=1}^{N} A_{i} \sin \left(\mathbf{k}_{i} \cdot \mathbf{x}+\omega_{i} t+\varphi_{i}\right) .
$$

This approach allows for easy control and variation of the properties of the advecting field, enabling us to get a deeper understanding of the basic mechanisms underlying the transport of passive particles which can them be applied to more realistic cases in a second step.

The motion of passive particles is described by the (normalized) $E \times B$ drift velocity

$$
v_{x_{1}}^{\mathrm{E}}=-\frac{\partial \phi(\mathbf{x}, t)}{\partial x_{2}}, \quad v_{x_{2}}^{\mathrm{E}}=\frac{\partial \phi(\mathbf{x}, t)}{\partial x_{1}} .
$$

Finite gyroradius effects are neglected here, but could be included in a straightforward fashion as has been done in previous publications [22, 32]. This (pseudo-)turbulent velocity 
field can be characterized by the Kubo number [33, 34]

$$
K \equiv \frac{V \tau_{c}}{\lambda_{c}}=\frac{\tau_{c}}{\tau_{\mathrm{fl}}}
$$

where $\tau_{c}$ and $\lambda_{c}$ denote, respectively, the autocorrelation time and length of the electrostatic potential, $V$ is the mean drift velocity, and $\tau_{\mathrm{fl}}$ is the mean time of flight for a distance of one correlation length. Therefore, the Kubo number can be interpreted as a measure for the trapping strength of a turbulent streamfunction.

The transport is characterized, as usual, by the second moment of the particle displacements,

$$
\left\langle x_{i}^{2}(t)\right\rangle \sim t^{\mu}
$$

where the angular brackets denote ensemble averaging. For $\mu=1$, one has standard diffusive behavior, while for $\mu<1$ and $\mu>1$, one has sub- and superdiffusive scaling, respectively. Since we will always find a transition to diffusive scaling for sufficiently long simulation times, it turns out to be convenient to plot the second moment in the form of a time dependent ('running') diffusion coefficient:

$$
D_{x_{i}}(t) \equiv \frac{1}{2} \frac{d}{d t}\left\langle x_{i}(t)^{2}\right\rangle
$$

In this context, the number of particles needs to be chosen such that $D(t)$ becomes sufficiently smooth. That number may be minimized via the 'time average' method described in Ref. [35]. Here, intermediate positions of the particles are reinterpreted as starting points of new trajectories.

In a pure isotropic and stochastic potential, transport obviously becomes diffusive as soon as the correlation time of the fluctuations is exceeded, since no memory exists. The introduction of streamers, i.e. structures which are elongated in the $x$ direction, only quantitatively changes the values of $D$, but preserves the diffusive character, since a complete decorrelation still occurs for $t>\tau_{c}$. [22] Interesting new regimes of transport can be expected only from additional coherent structures with much larger (or infinite) correlation times. In the following, we will modify the stochastic potential given by Eq. (1) by adding coherent flow effects. We hereby follow Ref. [22]; however, emphasis is now placed on the existence of an intermediate nondiffusive regime and the transition to standard diffusive transport for sufficiently long simulation times. 


\section{B. Poloidal shear flow effects}

In the case of ion temperature gradient (ITG) driven turbulence, the system usually spins up poloidal shear flows to fairly high amplitudes (see Ref. [36] and references therein). Thus it is interesting to investigate the effect of zonal flows on trace ion transport. As a model potential for the simulation of such flows, we choose

$$
\tilde{\phi}(x, y, t)=\phi(x, y, t)+A_{\mathrm{zf}} \sin \left(k_{\mathrm{zf}} x\right) .
$$

Here, $\phi$ represents an isotropic potential generated according to Eq. (1) with $N=10^{3}$ and $\lambda_{c}=1$, and the wave number of the zonal flow is chosen to be $k_{\mathrm{zf}}=0.78$. Varying $A_{\mathrm{zf}}$, the coherent component of the model potential can be changed. For comparison, we note that the root mean square of the stochastic component is $\sqrt{\left\langle\phi^{2}\right\rangle}=0.03$. In Fig. 1, the running diffusion coefficient as defined in Eq. (5) is plotted for different values of $A_{\mathrm{zf}}$. The Kubo number of the stochastic part has been chosen to be $K=5$ which is realistic (see Sec. III) and implies reasonably strong trapping events. In the pure stochastic potential $\left(A_{\mathrm{zf}}=0\right)$, we observe ballistic transport for small times which - after a short period of subdiffusion due to particle trapping - transitions to a diffusive regime as soon as the potential decorrelates (i.e. for $t \gtrsim \tau_{c} \approx 120$ ). With the increase of the zonal flow amplitude $A_{\mathrm{zf}}$, intermediate regimes of subdiffusion and superdiffusion emerge in the $x$ and $y$ direction, respectively. Qualitatively, the increase of transport in the direction of the flow and the decrease in the perpendicular direction are not difficult to understand, since the zonal flow term fundamentally changes the structure of the streamfunction, favoring open equipotential lines in the $y$ direction and suppressing them in the $x$ direction.

In this context, some interesting quantitative observations can also be made. First, the $y$ direction displays superdiffusive transport with a constant value $1<\mu<2$ for a relatively large time intervals, and second, for all values of $A_{\mathrm{zf}}$, there is a time scale beyond which the transport becomes diffusive. The first effect can be described phenomenologically in terms of a 'continous time random walk' (CTRW) model.[37, 38] Here, the subtle interplay between the stochastic fluctuations and the coherent component is represented by a purely probabilistic model built on waiting time and particle displacement distributions. Asymptotically, the Laplace transformation of the waiting time distribution can be modelled as $\tilde{\psi}(s)=1-A s^{\beta}$ with $0<\beta<1$, and the Fourier transform of the particle displacement distribution as $\tilde{f}(k)=1-B|k|^{\alpha}$ with $0<\alpha<2$. The exponent of the mean square displacement resulting 
from such a stochastic process can then be expressed as $\mu=2 \beta / \alpha$. Obviously, in the most simple approach, the particle motion underlying Fig. 1 can be modelled by slowly decaying distributions of the particle displacements in the $y$ direction and of the waiting time in the $x$ direction. Similar descriptions have already been applied successfully to other superdiffusive transport phenomena. $[12,17]$

Trying to understand the second effect mentioned above leads us to consider an additional physical mechanism. Comparing Fig. 1 with the PDF of the particle displacements in the $y$ direction plotted in Fig. 2 for $A_{\mathrm{zf}}=0.1$, we see that for intermediate times (when the transport is superdiffusive), there are two peaks in the PDF - representing the bulk of particles which is advected ballistically up and down the $y$ direction with the mean shear flow velocity. The peak at $y=0$ stands for the particles being trapped by vortices at the combs of the potential. The interaction between trapping caused by the stochastically evolving vortices and the free poloidal motion due to the strong zonal flow component leads to a slowly decaying particle displacement distribution and to an exponent $\mu>1$. Furthermore, comparing Fig. 1 and Fig. 2, we notice that the transition to the diffusive regime occurs when the ballistic peaks of the PDF have just vanished. Fig. 3 sheds some more light onto this effect. We observe that for $t \lesssim t_{1} \sim 2000$ - i.e. during the time span for which the transport is superdiffusive in the $y$ direction and subdiffusive in the $x$ direction - the particle distribution is trapped inside the individual shear flow 'channels' $\left(-\pi / k_{\mathrm{zf}}<x<\pi / k_{\mathrm{zf}} \approx 4\right)$. For larger times $\left(t \gtrsim t_{1}\right)$, we then find that particles may move into a neighboring structure. This means that the direction of the flow is reversed, and a randomization takes place.

In order to make this transition more transparent, we measured the distribution of the first passage time of the particles, i.e. the time interval which particles need to leave a given flow channel and move from an upward to a downward flow or vice versa. This distribution is plotted for $A_{\mathrm{zf}}=0.1$ in Fig. 4. It is clear that if a finite transition probability between the flow structures exists - which will always be the case it a perturbation in form of time dependent fluctuations is introduced - the waiting time distribution will fall off exponentially. For such a function, the first moment exists, and given the fact that we can characterize the particle displacements in the $x$ direction as discrete steps of size $\Delta x=\pi / k_{\mathrm{zf}}$, this in turn means that the transport becomes diffusive.[37] Consequently, the dynamics on large time scales can be modeled by an ordinary random walk with $D \sim \Delta x^{2} /(2 \Delta t)$, where $\Delta t$ is defined as the e-folding length of the passage time distribution. With $\Delta t \approx 9500$ and 
$\Delta x \approx 4$ we find $D \approx 0.00085$ which favorable agrees with the simulated diffusion coefficient for $A_{\mathrm{zf}}=0.1$ plotted in Fig. 1. In terms of the CTRW model, this situation corresponds to a 'truncated waiting time distribution'. The first passage time of the test particles plotted in Fig. 4 shows a sharp increase of $\Psi(t)$ followed by a flat regime of $\Psi(t)$ for intermediate times $\left(t \lesssim t_{1}\right)$ and an exponential decay for $t \gtrsim t_{1}$. Using the CTRW mechanism, such a distribution reproduces the observed diffusivities.[39] In particular, we observe that the time scale $t_{1}$ inferred from Fig. 4 corresponds to the time $t_{1}$ in Fig. 1 . It is clear from this discussion that the intermediate time interval which is characterized by super- and subdiffusion can grow indefinitely as the zonal flow term gets stronger and stronger.

In previous publications, anomalous transport behavior has sometimes been attributed to the existence of 'chaotic jets', i.e. special paths in a generally chaotic environment exhibiting a large stickiness of the advected particles as well as a coherent, ballistic motion.[17-19] As an indicator, an accumulation of Lyapunov exponents near zero was identified. In Fig. 5, the distribution of Lyapunov exponents is plotted for different values of $A_{\mathrm{zf}}$. These quantities are calculated via [18]

$$
\sigma_{L}=T^{-1} \ln (\delta / \epsilon)
$$

where $T$ is the time two particles remain within a distance smaller than $\delta$, and $\epsilon$ is the initial separation of the two particles. One typically assumes $\delta \gg \epsilon$ with both quantities chosen to be rather small. We observe, however, that even for very large zonal flow amplitudes [recall that the stochastic component of Eq. (6) is $\sqrt{\left\langle\phi^{2}\right\rangle}=0.03$ ], there is no accumulation of Lyapunov exponents near $\sigma_{L}=0$. This behavior in turn corresponds to the exponential decay of the PDF of the 'sticking time' $T$ which we observed for all the cases displayed in Fig. 5. Such a decay is a normal consequence of the 'clump effect',[20] and does not depend on the existence of any special structure. Simply said, two particles starting in a close neighborhood separate when they encounter a hyperbolic fixed point. Since in time dependent potentials this is a stochastic process with a given probability, the 'sticking time' will naturally exhibit an exponential decrease. So although strong coherent structures are present in this model potential, no chaotic jets can be observed. 


\section{Poloidal drift effects}

Generally, all microinstabilities in toroidal magnetoplasmas exhibit drifts in the poloidal direction. While ion temperature gradient (ITG) modes tend to drift in the ion diamagnetic direction, electron temperature gradient (ETG) modes and trapped electron modes usually drift in the electron diamagnetic direction. Now, in Ref. [22] it was shown that homogeneous poloidal drifts may have the same effect on transport as poloidal shear flows. In that paper, a 'drifting potential' $\phi_{\mathrm{dr}}$ was defined as a (fluctuating or static) potential whose structures move in the poloidal $(y)$ direction with a constant drift velocity $v_{\mathrm{dr}}$. Denoting again the isotropic potential created via Eq. (1) by $\phi(x, y, t)$, we thus have

$$
\phi_{\mathrm{dr}}(x, y, t) \equiv \phi\left(x, y-v_{\mathrm{dr}} t, t\right)
$$

In order to understand the effect of such a homogeneous drift, it is useful to perform a Galilei transformation to a reference frame moving in the $y$ direction with velocity $v_{\mathrm{dr}}$. Quantities referring to this co-moving coordinate system will be denoted with a prime. Using Faraday's law of induction (in the limit of non-relativistic velocities) this transformation leads to a electrostatic potential

$$
\phi_{\mathrm{dr}}^{\prime}(x, y, t)=\phi_{\mathrm{dr}}\left(x, y+v_{\mathrm{dr}} t, t\right)-v_{\mathrm{dr}} x=\phi(x, y, t)-v_{\mathrm{dr}} x
$$

For the particle motion we then find the (trivial) relation $\mathbf{v}_{E}^{\prime}=\mathbf{v}_{E}-v_{\mathrm{dr}} \mathbf{e}_{y}$. So in the comoving frame, which is more easy to access, the law of induction produces an additional electric field which is responsible for the differences compared to a non-drifting potential. The second term on the right-hand side of Eq. (9) lends the potential a completely new structure. Qualitatively, the latter is comparable to that of a zonal flow potential defined by Eq. (6). As we will see shortly, the particle transport indeed exhibits the same behavior as found in the previous section. This includes, in particular, the supression of transport in the $x$ direction and its increase in the $y$ direction, going along with sub- or superdiffusive transport for intermediate time scales.

Fig. 6 shows the running diffusion coefficient for different drift velocities $v_{\mathrm{dr}}$. It can clearly be seen that the effect of the poloidal drift is similar to the effect of zonal flows (compare to Fig. 1), in both the radial and the poloidal direction. However, the additional term in Eq. (9) is not periodic now, but corresponds to an infinite ramp. This makes the transport 
suppression in the $x$ direction stronger. For large times, we again find a transition to diffusive behavior. The modelling in terms of a random walk model seems to be difficult at first, since in contrast to the zonal flow potential, no reference length scale is given explicitly. On the other hand, it is possible to define an intrinsic length scale via the maximum excursion of an equipotential line in the $x$ direction, $x_{\max } \approx 2 \phi_{\max } / v_{\mathrm{dr}} \approx 2 V \lambda_{c} / v_{\mathrm{dr}}$ (here, $\lambda_{c}$ is the correlation length of $\phi$, and $V$ is the average $E \times B$ drift velocity). For the given potential and $v_{\mathrm{dr}}=0.05$, we find $x_{\max } \approx 2$. The relavance of this limit can clearly be observed in the PDF of Fig. 7 for small times, whereas for larger times, the transport becomes diffusive. So the effect is basically the same as in the zonal flow case. In Fig. 8, the PDF is displayed for the $y$ direction. Again, we observe a similar behavior as in the zonal flow case (Fig. 2), but we now have a single peak of trapped particles moving upwards with the drift velocity and a bulk of particles moving downwards on open equipotential lines, whereas in the former case, we found two peaks moving upwards and downwards within a given zonal flow channel. Similarly, the transition to a normal diffusive behavior is not caused by the transition to a flow with reversed flow direction, but by the transition from trapped particles advected with the diamagnetic drift to free particles moving against the drift and vice versa. A similar, though more qualitative study of the interplay between free and trapped advected particles can be found in Ref. [40].

In order to further investigate the time evolution of the PDFs shown in the last two subsections, a plot of the kurtoses for all the discussed cases is shown in Fig. 9. We define the kurtosis as

$$
\gamma_{2}=\left\{\frac{1}{N} \sum_{i=1}^{N}\left[\frac{x_{i}-\bar{x}}{\sigma}\right]^{4}\right\}-3,
$$

where $N$ is the number of test particles, $x_{i}$ is the displacement of a single particle from its origin, $\bar{x}$ is the mean value of $x_{i}$ and $\sigma$ is the mean square displacement. As is well known, Gaussian distributions are characterized by $\gamma_{2}=0$, whereas $\gamma_{2}>0\left(\gamma_{2}<0\right)$ indicates a slower (faster) than Gaussian decay. The kurtoses in Fig. 9 are calculated for the same data plotted as PDF in Figs. 2, 3, 7 and 8. We clearly observe the transition $\gamma_{2} \rightarrow 0$ for large times. Interestingly, the kurtosis for the $x$ direction for a drifting potential is close to zero for all times despite the fact that there exists a spatial limit (see Fig. 7). We can explain this observation by the fact that this limit is not a 'hard' one like in the zonal flow case, but rather a 'soft' one. 
Hence we have demonstrated that the existence of large coherent components in a fluctuating potential - represented here by a static zonal flow and a constant drift velocity - can lead to extended regimes of anomalous diffusion for intermediate times, but always shows a transition to the diffusive regimes for large times. In the following, we show that this simple model displays a good correspondence to transport in realistic potentials as described by nonlinear gyrokinetics, and it will turn out that the above interpretations for the simple models carry over to the more realistic ones.

\section{DIFFUSION IN REALISTIC TURBULENT FIELDS}

Until now, we have studied the transport of passive tracers in electrostatic potentials which were created by superposing a large number of plane waves. Applying this method, we where able to model and analyze the influence of zonal flows and diamagnetic drifts on

the transport. The variation of the strength of those additional effects enabled us to study the regime of anomalous diffusion for intermediate times and the conditions for a transition to normal diffusion. Keeping these results in mind, we now want to consider the transport behavior of test particles in realistic electrostatic potentials as they occur for ITG and TEM turbulence. As we will see, these two cases exhibit the same effects as discussed in the previous section.

For this purpose, we use simulation data obtained with the nonlinear gyrokinetic code GENE. [25, 26] The electrostatic potential in a perpendicular plane on the low-field side is written out frequently and then subjected to post-processing. For spatial interpolation, a simple bicubic scheme is used. (In order to test its accuracy, comparisons with Fourier interpolation - which is the most exact interpolation method - have been performed for a few test cases, but no significant differences were found.) We note that from now on, perpendicular length scales are normalized with respect to $\rho_{s}=c_{s} / \Omega_{i}$ where $c_{s}=\left(T_{e} / m_{i}\right)^{1 / 2}$ is the ion acoustic velocity and $\Omega_{i}$ the ion gyrofrequency, whereas time scales are normalized with respect to $L_{\perp} / c_{s}$ where $L_{\perp}$ is a scale length of the background profiles (see, e.g., Ref. [26]). In this context, we would also like to note that GENE uses periodic radial boundary conditions (keeping the average background gradients fixed), and that the simulation box sizes were chosen about one order of magnitude larger than the correlation lengths of the turbulence. Therefore, finite size effects do not play a role. 
First, we study the test particle transport in ITG turbulence. Here, the so-called Cyclone base case parameter set as described in Ref. [41] is used. A typical contour plot of the electrostatic potential in the outboard midplane is shown in Fig. 10. The zonal flow structures are very prominent. As one may expect from this figure, the running diffusion coefficient exhibits a distinct regime of superdiffusion in the $y$ direction for intermediate times, together with a (less distinct) regime of subdiffusion in the $x$ direction (Fig. 11). The curves show a close correspondence to the cases with high zonal flow amplitude in Fig. 1. In addition, the PDFs of the particle displacements for a number of different times are given in Figures 12 and 13. Their shape is again comparable to the PDFs plotted in Figures 3 and 2. Especially, the threshold of $\Delta x=\pi / k_{\mathrm{zf}} \approx 40$ can be observed in Fig. 12, and the disappearance of the ballistic peaks of the $y$-PDF corresponds again to the transition to diffusive transport.

Second, the transport of test particles in TEM turbulence is studied (for details, see Ref. [22]). Here, zonal flows tend to be rather weak [26], such that diamagnetic drift effects will be more important. A plot of the running diffusion coefficient for this case can be found in Ref. [22]. Therein, the correspondence to the studies employing self-created potentials has already been demonstrated. However, since the diamagnetic drift velocity is relatively small in that case, the PDFs do not exhibit a clear threshold effect in the $x$ direction and the ballistic peaks in the $y$ direction, and their shape is quite close to a Gaussian even for small times.

In Fig. 13, the kurtoses of the particle PDFs are plotted for the ITG as well as for the TEM potential versus time. Similar to Fig. 9, the transition to a Gaussian distribution, expressed by $\gamma_{2} \rightarrow 0$ for large times, can be observed. Therefore, one may conclude that the studies done in Sec. II as well as the corresponding results may be considered quite realistic. In the presence of sufficiently strong poloidal flows, sheared or homogeneous, one may expect to find superdiffusion in the $y$ direction and subdiffusion in the $x$ direction of an intermediate time span which grows as the amplitude of the coherent flow component increases.

\section{SUMMARY AND CONCLUSIONS}

The main goal of the present work was to investigate the behavior of test particles in realistic turbulent fields, focussing on the question whether and under what conditions the 
transport may become 'anomalous', i.e. super- or subdiffusive. For simplicity, we have restricted to electrostatic fluctuations and two dimensions as far as the particle trajectories are concerned. Nevertheless, we have used electrostatic potentials taken from three (space) dimensional gyrokinetic simulations with the GENE code. Here, ion temperature gradient and trapped electron mode turbulence cases were chosen, both exhibiting coherent flow components in addition to stochastic fluctuations. In addition, we considered potentials which were generated by superposing a large number of random waves. The variation of the strength of the coherent part (given by the zonal flow amplitude or the drift velocity) enabled us to gain a basic understanding of several transition phenomena.

While in the presence of stochastic fluctuations, one always observes that the particle transport eventually becomes diffusive [in contrast to some previous studies which dealt with less realistic models, observing anomalous diffusion for arbitrarily long times], sufficiently strong poloidal flows, sheared or homogeneous, tend to induce superdiffusion in the $y$ direction and subdiffusion in the $x$ direction over an intermediate time span. The latter grows as the amplitude of the coherent flow component increases, and it may, in fact, become much larger than the correlation time of the fluctuations. Thus, if one is interested in transitional - not steady-state - phenomena, the effects described in the present work are likely to be of relevance for the cross-field transport induced by microturbulence.

\section{Acknowledgments}

We thank T. Görler, M. Püschel and F. Merz for providing simulation data obtained with the GEnE code. We are also grateful to R. Friedrich for fruitful discussions concerning the probabalistic description of the observed transport processes. 
[1] F. W. Perkins, C. W. Barnes, D. W. Johnson, et al., Phys. Fluids B 5, 477 (1993).

[2] K. W. Gentle, R. V. Bravenec, G. Cima, et al., Phys. Plasmas 2, 2292 (1995).

[3] J. D. Callen and M. W. Kissick, Plasma Phys. Contr. Fusion 39, B173 (1997).

[4] B. A. Carreras, B. van Milligen, M. A. Pedrosa, et al., Phys. Rew. Lett. 80, 4438 (1998).

[5] B. A. Carreras, B. P. van Milligen, M. A. Pedrosa, et al., Phys. Plasmas 6, 1885 (1999).

[6] G. M. Zaslavsky, M. Edelman, H. Weitzner, B. Carreras, G. McKee, R. Bravenec, and R. Fonck, Phys. Plasmas 7, 3691 (2000).

[7] D. R. Baker, C. M. Greenfield, K. H. Burrell, et al., Phys. Plasmas 8, 4128 (2001).

[8] B. A. Carreras, V. E. Lynch, D. E. Newman, and G. M. Zaslavsky, Phys. Rev. E 60, 4770 (1999).

[9] B. A. Carreras, D. Newman, V. E. Lynch, and P. H. Diamond, Phys. Plasmas 3, 2903 (1996).

[10] J. A. Mier, L. Garcia, and R. Sanchez, Phys. Plasmas 13, 102308 (2006).

[11] B. A. Carreras, V. E. Lynch, and G. M. Zaslavsky, Phys. Plasmas 8, 5096 (2001).

[12] D. del-Castillo-Negrete, B. A. Carreras, and V. E. Lynch, Phys. Plasmas 11, 3854 (2004).

[13] B. P. van Milligen, R. Sanchez, and B. A. Carreras, Phys. Plasmas 11, 2272 (2004).

[14] B. P. van Milligen, B. A. Carreras, and R. Sanchez, Phys. Plasmas 11, 3787 (2004).

[15] ITER Physics Basis, Nucl. Fusion 39, 2137 (1999).

[16] R. Balescu, Aspects of Anomalous Transport in Plasmas, p. 418 (IoP Publishing, Bristol, 2005).

[17] V. V. Afanasiev, R. Z. Sagdeev, and G. M. Zaslavsky, Chaos 1, 143 (1991).

[18] X. Leoncini and G. M. Zaslavsky, Phys. Rev. E 65, 046216 (2002).

[19] X. Leoncini, O. Agullo, S. Benkadda, and G. M. Zaslavsky, Phys. Rev. E 72, 026218 (2005).

[20] Ref. [16], Ch.13.

[21] S. V. Annibaldi, G. Manfredi, and R. O. Dendy, Phys. Plasmas 9, 791 (2002).

[22] T. Hauff and F. Jenko, $E \times B$ advection of trace ions in tokamak microturbulence, Phys. Plasmas in print (2007).

[23] D. del-Castillo-Negrete, Phys. Fluids 10, 576 (1998).

[24] D. del-Castillo-Negrete, Phys. Plasmas 7, 1702 (2000).

[25] F. Jenko, W. Dorland, M. Kotschenreuther, and B. N. Rogers, Phys. Plasmas 7, 1904 (2000). 
[26] T. Dannert and F. Jenko, Phys. Plasmas 12, 072309 (2005).

[27] T. S. Hahm, Phys. Fluids 31, 2670 (1988).

[28] T. S. Hahm, W. Lee, and A. Brizard, Phys. Fluids 31, 1940 (1988).

[29] A. Brizard, Phys. Fluids B 1, 1381 (1989).

[30] R. E. Waltz, J. M. Candy, and M. N. Rosenbluth, Phys. Plasmas 9, 1938 (2002).

[31] J. Candy, R. E. Waltz, and W. Dorland, Phys. Plasmas 11, L25 (2004).

[32] T. Hauff and F. Jenko, Phys. Plasmas 13, 102309 (2006).

[33] R. Kubo, J. Math. Phys. 4, 174 (1963).

[34] M. Vlad, F. Spineanu, J. H. Misguich, J. D. Reuss, R. Balescu, K. Itoh, and S. I. Itoh, Plasma Phys. Control. Fusion 46, 1051 (2004).

[35] J. H. Reuss, and J. D. Misguich, Phys. Rev. E 54, 1857 (1996).

[36] P.H. Diamond, S.-I. Itoh, K. Itoh, and T.S. Hahm, Plasma Phys. Control. Fusion 47, R35 (2005).

[37] E. W. Montroll and G. H. Weiss, J. Math. Phys. 6, 167 (1965).

[38] R. Metzler and J. Klafter, Phys. Rep. 339, 1 (2000); J. Phys. A 37, R161 (2004).

[39] M. Dentz, A. Cortis, H. Scher, and B. Berkowitz, Adv. Water Resour. 27, 155 (2004).

[40] V. Naulin, A. H. Nielsen, and J. Juul Rasmussen, Phys. Plasmas 6, 4575 (1999).

[41] A. M. Dimits, G. Bateman, M. A. Beer, et al., Phys. Plasmas 7, 969 (2000). 


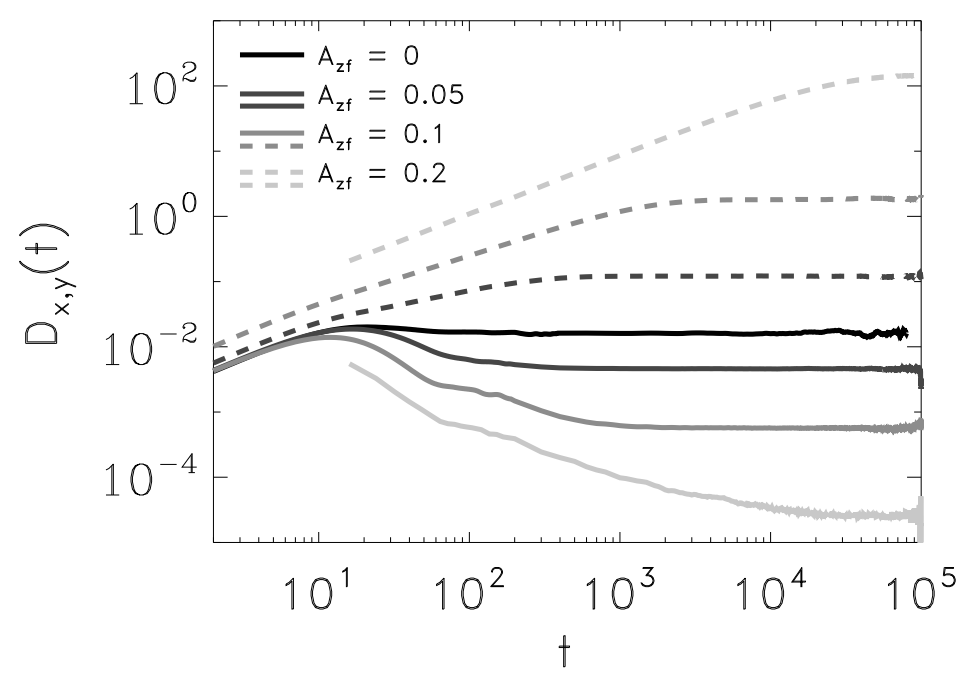

FIG. 1: Running diffusion coefficient $D(t)$ for different zonal flow amplitudes [Eq. $(6), K=5$ ]. The solid lines denote the $x$ direction, the dashed lines the $y$ direction. In the $y$ direction, a superdiffusive transitional regime is found for intermediate times. In that regime, we find $\mu \approx 1.5$ for $A_{\mathrm{zf}}=0.05, \mu \approx 1.7$ for $A_{\mathrm{zf}}=0.1$, and $\mu \approx 1.9$ for $A_{\mathrm{zf}}=0.2$. 


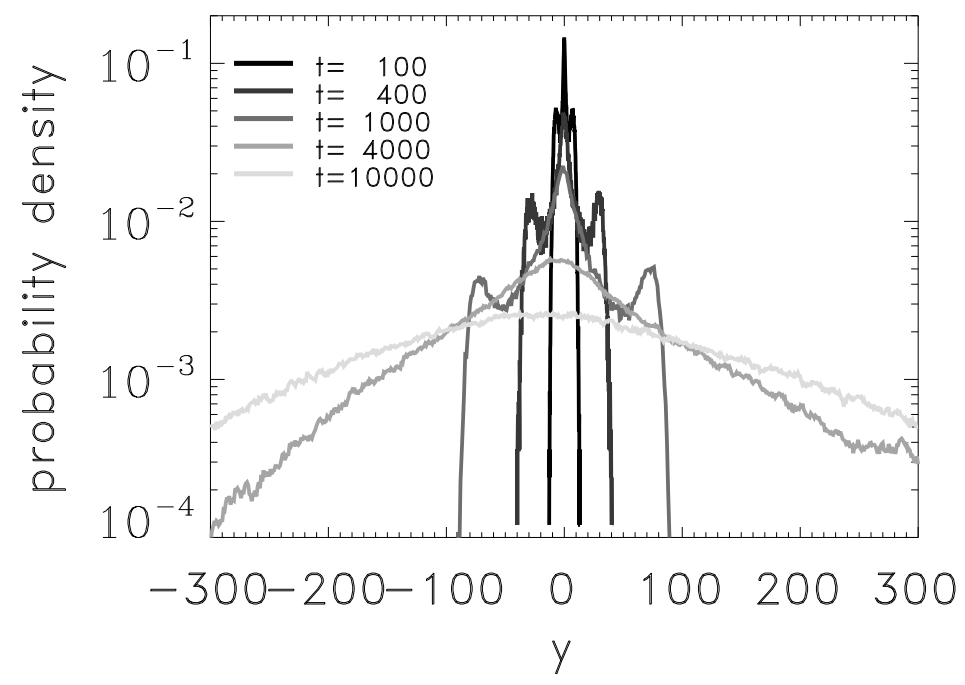

FIG. 2: PDF of the particle displacements in the $y$ direction for $K=5$ and $A_{\mathrm{zf}}=0.1$ for different times. [Note that $\tau_{c} \approx 100$.] For small times, the superdiffusive advection of the tracers with the shear flow can be observed for positive and negative directions. For $t>t_{1} \approx 2000$, transitions between different zonal flow channels occur, transforming the distribution into a Gaussian one.

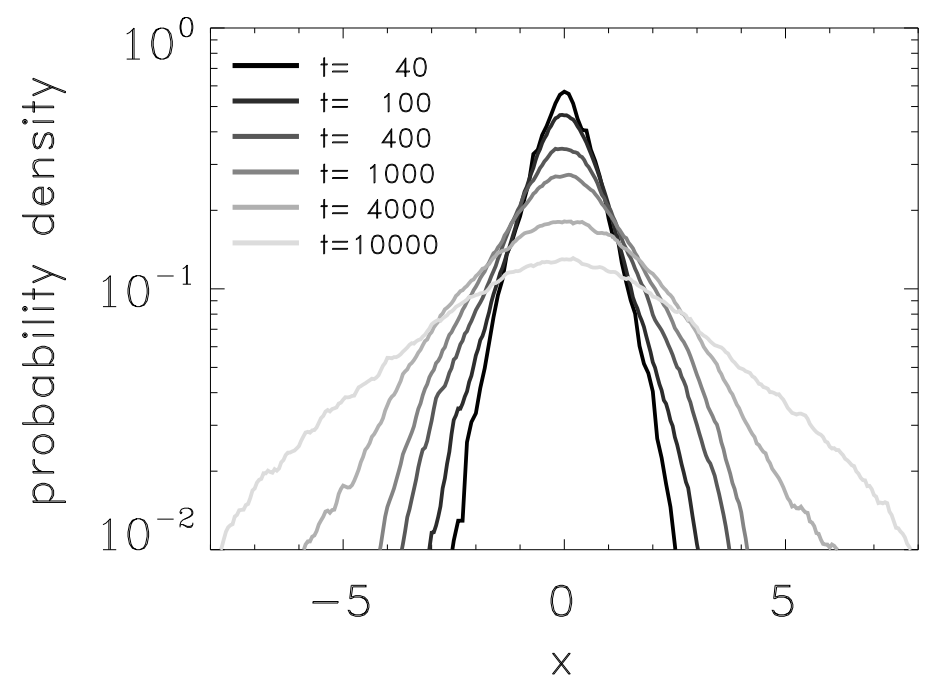

FIG. 3: Same as Fig. 2, but for the $x$ direction. For times $t<t_{1} \approx 2000$, particles are trapped inside a shear layer of the width $\pi / k_{\mathrm{zf}} \approx 4$. For larger times, a significant number of transitions into neighboring layers occur. 


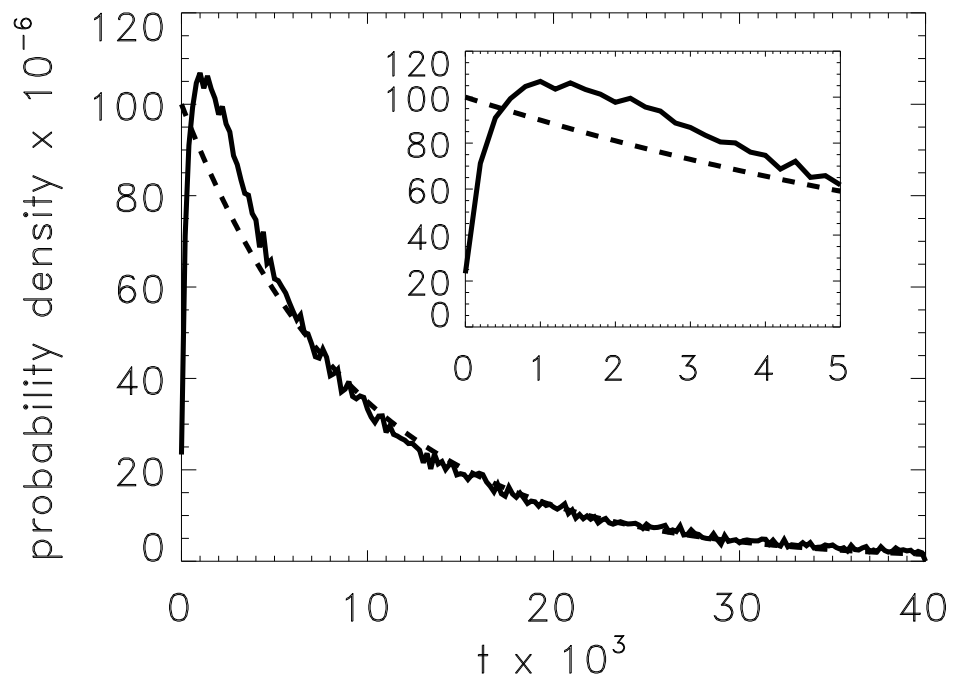

FIG. 4: First passage time of a particle to move from an up- into a downward flow or vice versa for $A_{\mathrm{zf}}=0.1$. Solid line: PDF of the first passage time from the simulation. Dashed line: $0.0001 \exp (-t / 9500)$.

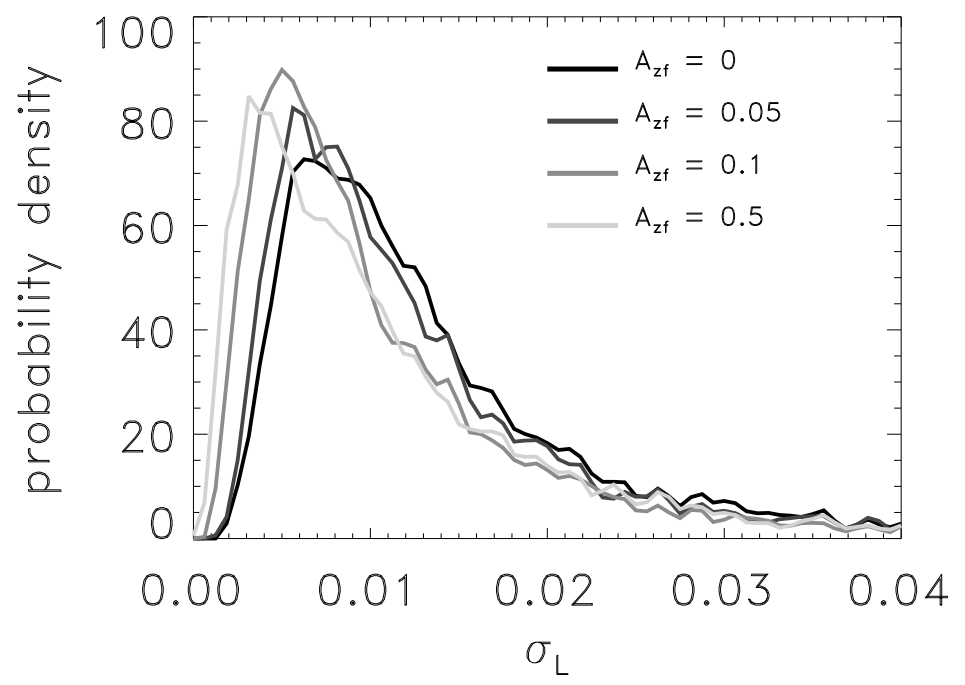

FIG. 5: PDF of the Lyapunov exponent $\sigma_{L}=T^{-1} \ln (\delta / \epsilon)$ for different values of $A_{\mathrm{zf}}$. Here, $\delta=0.1$ and $\epsilon=0.001$. No accumulation near $\sigma_{L}=0$ is observed, even for very large zonal flow amplitudes. 


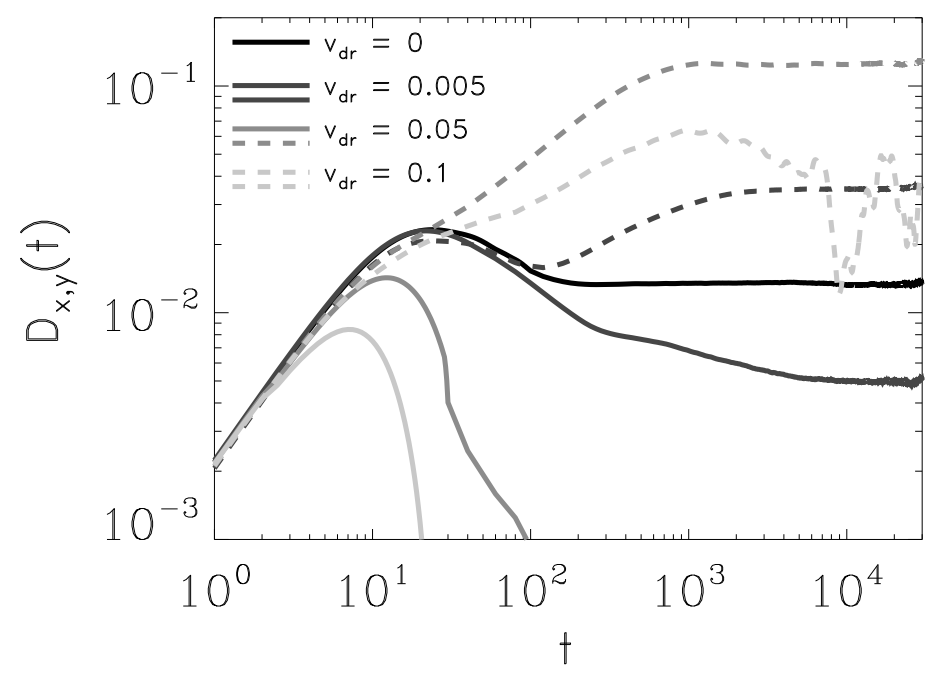

FIG. 6: Running diffusion coefficient $D(t)$ for different drift velocities [Eq. (8), $K=20$ ]. The solid lines denote the $x$ direction, the dashed lines the $y$ direction. In the $y$ direction, a superdiffusive transitional regime is found for intermediate times. In that regime, we find $\mu \approx 1.35$ for $v_{\mathrm{dr}}=0.005$, $\mu \approx 1.55$ for $v_{\mathrm{dr}}=0.05$, and $\mu \approx 1.39$ for $v_{\mathrm{dr}}=0.1$. The saturation values for the $x$ direction outside the range of the figure are $D_{x}=10^{-4}$ for $v_{\mathrm{dr}}=0.05$ and $D_{x}=5 \times 10^{-6}$ for $v_{\mathrm{dr}}=0.1$. 


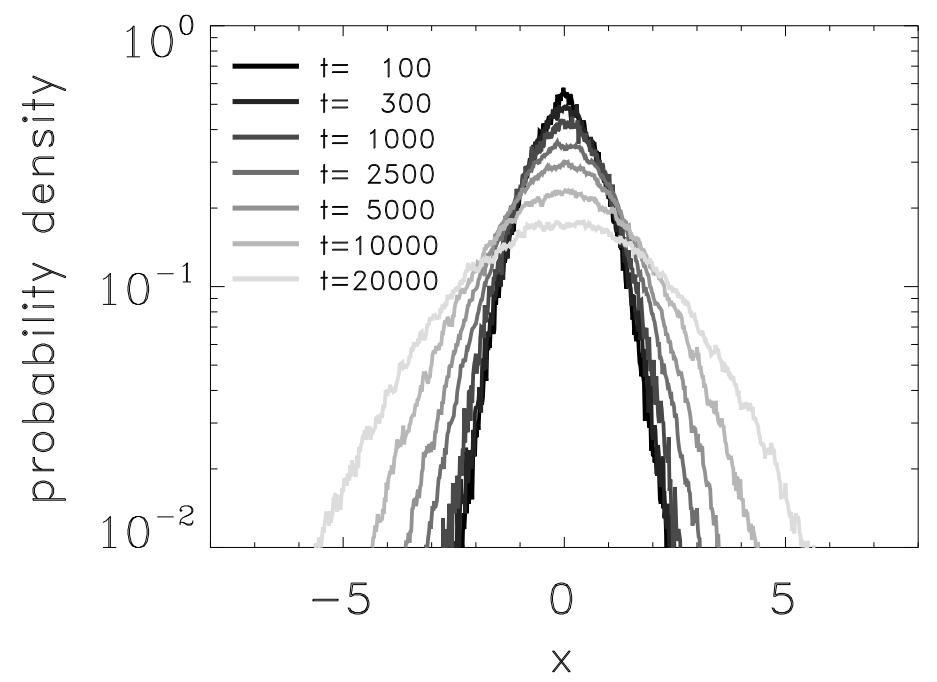

FIG. 7: PDF of the particle displacements in the $x$ direction for $K=20, v_{\mathrm{dr}}=0.05$ for different times. Note that $\tau_{c} \approx 100$. For times $t<2000$ particles are trapped inside a threshold of the width $x_{\max } \approx 2 V \lambda_{c} / v_{\mathrm{dr}} \approx 2$. For larger times, a significant number of transitions into neighboring layers occur.

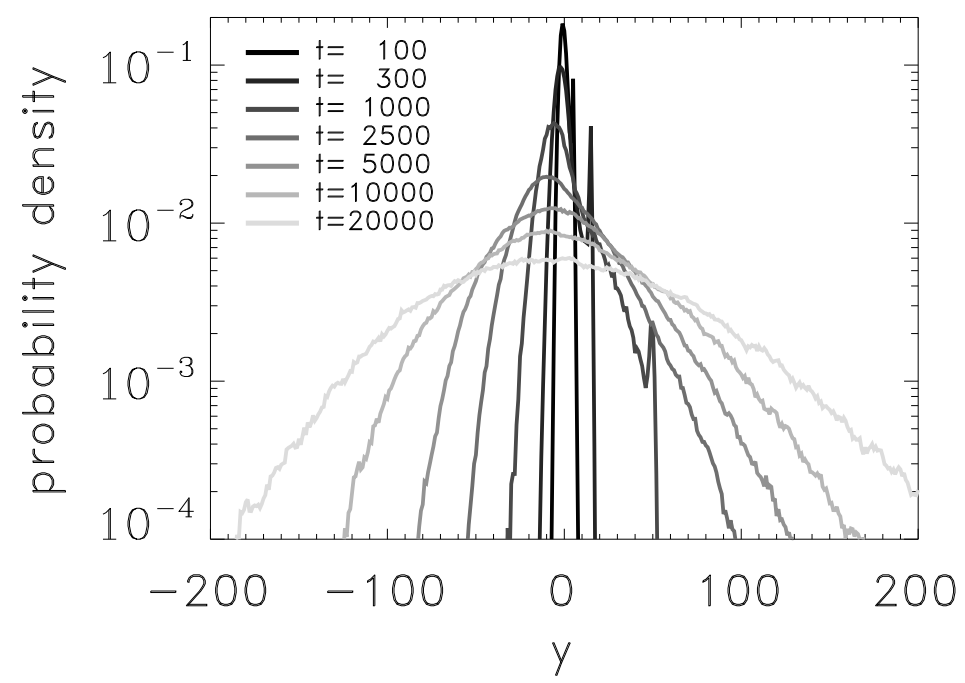

FIG. 8: Same as Fig. 7, but for the $y$ direction. For small times, peaks can be observed at $y=v_{\mathrm{dr}} t$, displaying the motion of trapped particles with the diamagnetic flow in the positive $y$ direction. For $t>1000$, transitions between the trapped upward-moving and the free downward-moving particles evolve, transforming the distribution into a Gaussian one. 


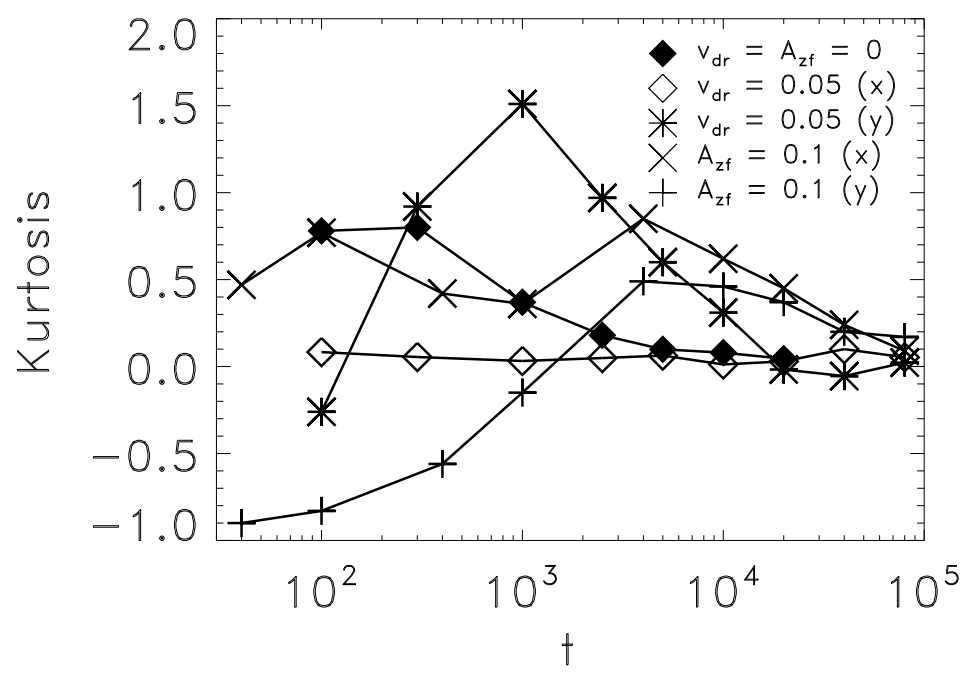

FIG. 9: Kurtoses for the PDFs shown in Figs. 2, 3, 7 and 8. The kurtoses cleary approach zero for large times, indicating the transition to a Gaussian distribution.

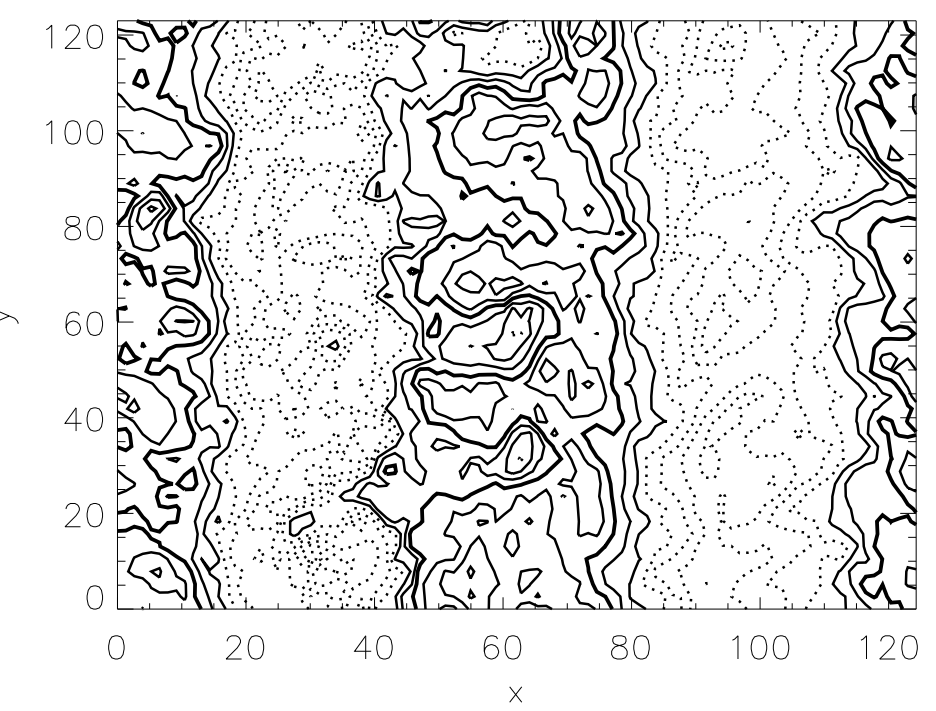

FIG. 10: Contours of the ITG electrostatic potential ('Cyclone base case'). Positive contours are drawn with solid lines, negative contours with dashed lines. Strong zonal flows are present, forming a dominant coherent background. 


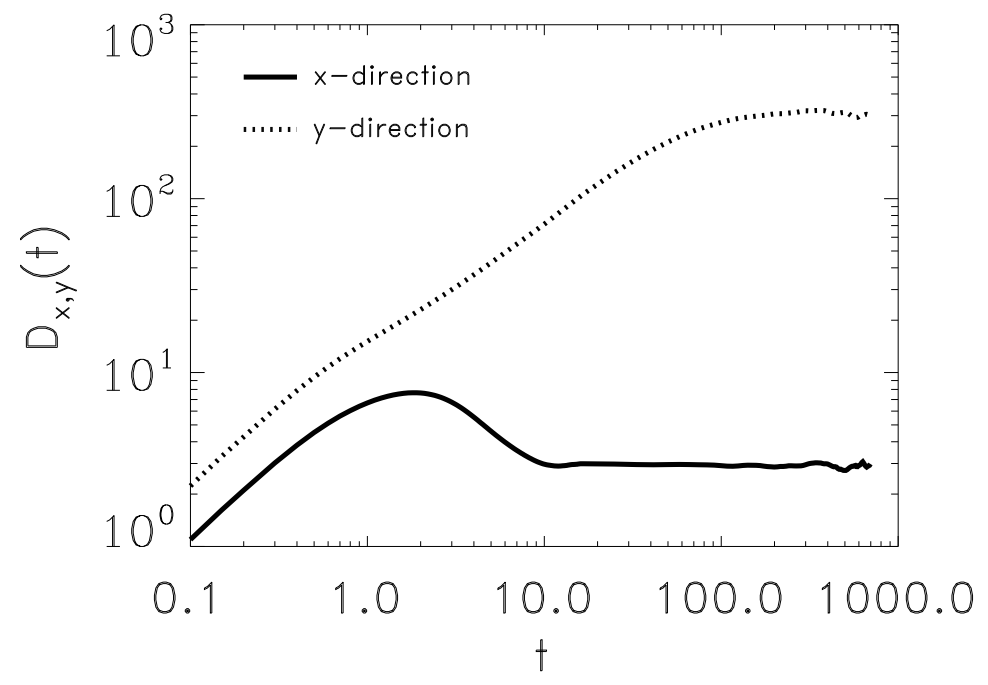

FIG. 11: Running diffusion coefficient $D(t)$ for the ITG electrostatic potential plotted in Fig. 10 $(K \approx 7)$. The curves are comparable to the ones obtained by the self-created potential (Fig. 1$)$. For the superdiffusive transition regime in the $y$ direction we find $\mu \approx 1.72$ for intermediate times.

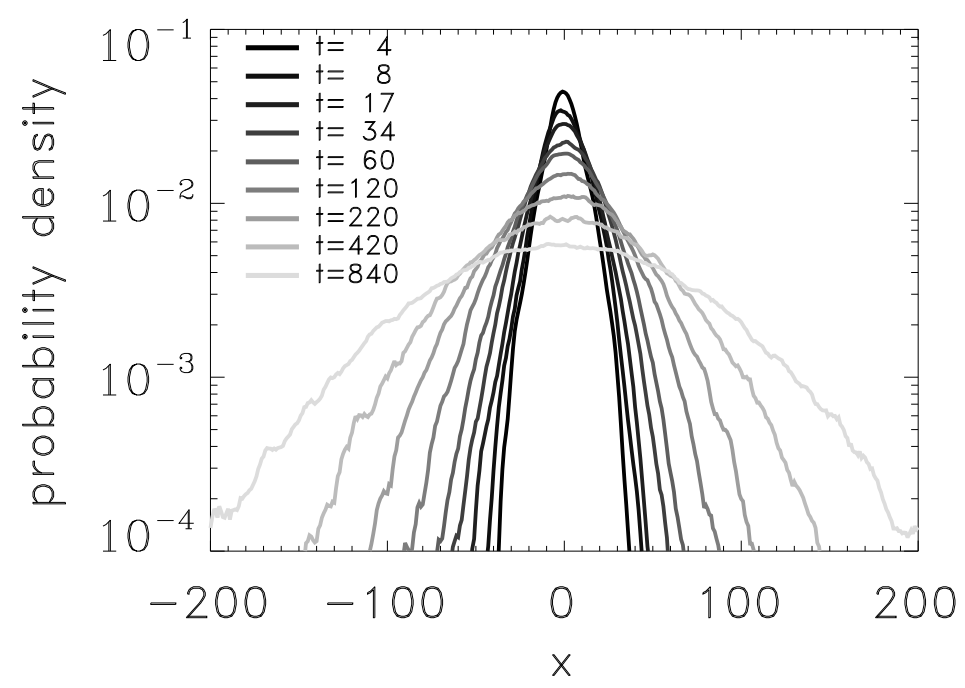

FIG. 12: PDF of the particle displacements in the $x$ direction for the ITG potential for different times. The behavior is comparable to the one shown in Fig. 3. 


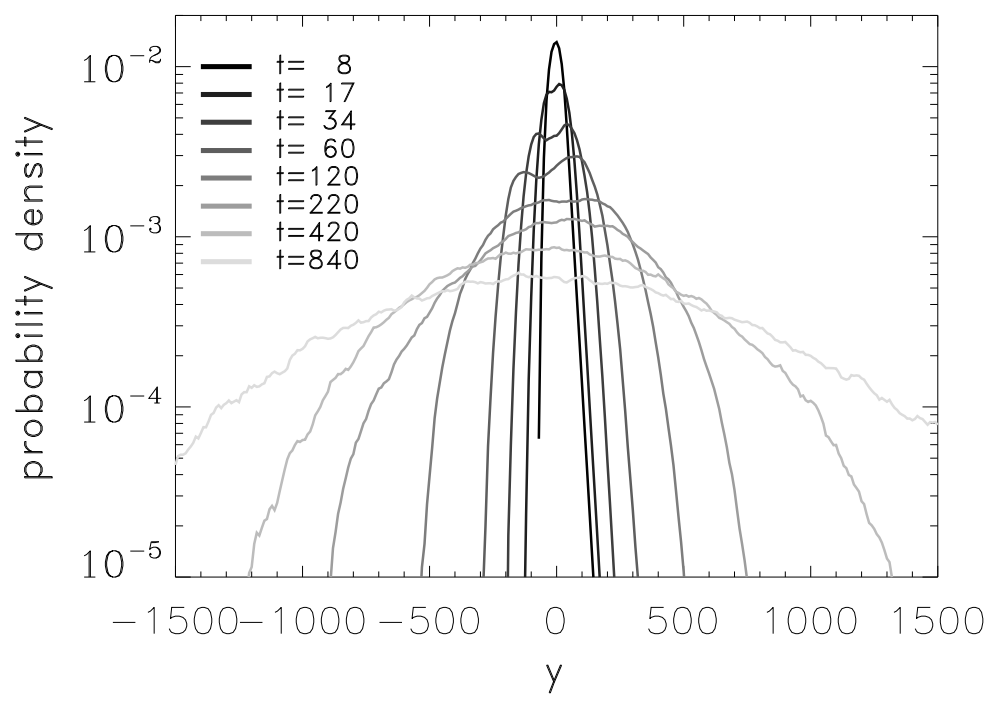

FIG. 13: PDF of the particle displacements in the $y$ direction for the ITG potential for different times. The behavior is comparable to the one shown in Fig. 2.

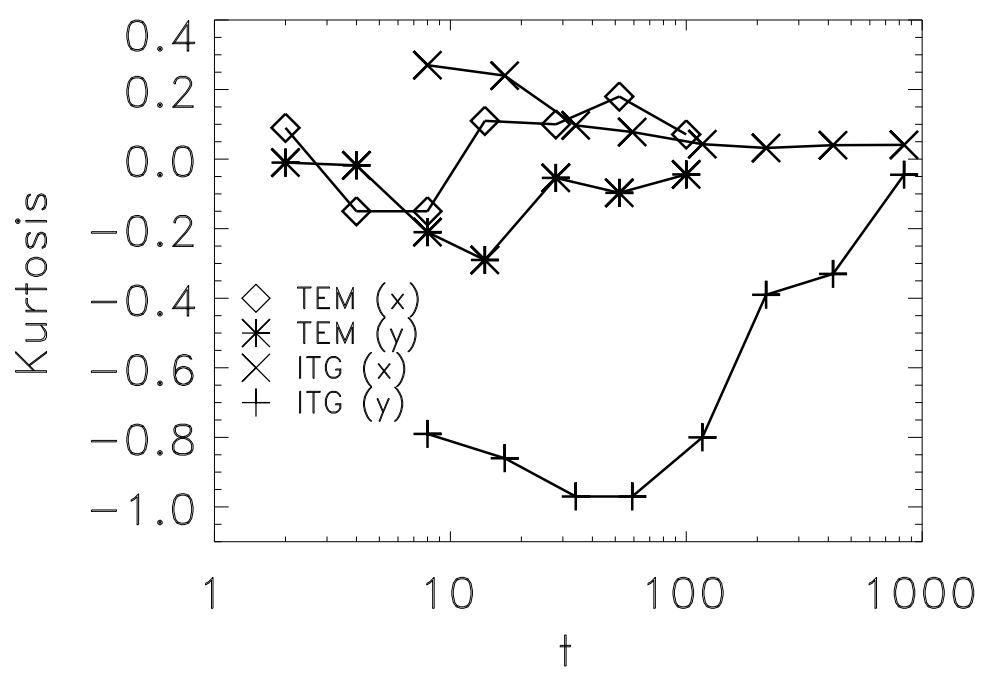

FIG. 14: Kurtoses for the PDFs shown in Figs. 12 and 13, and for the TEM potential. Again, the kurtoses cleary approach zero for large times, indicating the transition to a Gaussian distribution. 